\title{
A Cross-Sectional Study of Blood Donors' Psychological Characteristics over 8 Weeks
}

\author{
Max Esefeld ${ }^{a}$ Ariane Sümnig ${ }^{a, b} \quad$ Ulf Alpen ${ }^{a}$ Hans Jörgen Grabe ${ }^{c}$ \\ Andreas Greinacher ${ }^{\mathrm{a}}$ \\ alnstitut für Immunologie und Transfusionsmedizin, Universitätsmedizin Greifswald, Greifswald, Germany; \\ ${ }^{b}$ MVZ Labor Greifswald GmbH, Greifswald, Germany; 'Klinik und Poliklinik für Psychiatrie und Psychotherapie, \\ Universitätsmedizin Greifswald, Greifswald, Germany
}

\section{Keywords}

Donor research $\cdot$ Effects of blood donation $\cdot$ Mood regulation

\begin{abstract}
Background: Previous studies suggest that blood donation impacts blood donors' psychological state, with either positive or negative effects, such as feeling more energetic or more exhausted. It has not yet been described how long these effects last. Materials and Methods: This prospective cohort study consisted of a qualitative and a quantitative part: (1) Psychological characteristics which changed after blood donation were identified by structured interviews of regular whole blood donors $(n=42)$. Based on this, a questionnaire addressing 7 psychological dimensions was established. (2) The psychological state of 100 blood donors was assessed after blood donation by applying the questionnaire 15-30 min before and during donation, as well as 15-30 min, 6 h, 24 h, 72 h, 1 week, and 8 weeks after donation. The resulting changes were summarized to a score. Furthermore, potential correlations of the score with pre-donation blood pressure, hemoglobin, or body mass index were calculated. Results: Seven items were identified which changed in at least $25 \%$ of blood donors (mood, concentration, satisfaction, resilience, spirit of initiative, physical well-being, energy level). In the 100 blood donors, the well-being score increased (positive effects, $n=23$ ), showed minor changes ( $n$ $=53$ ), or decreased (negative effects, $n=24$ ). The positive effects lasted for about 1 week and the negative effects for 3
\end{abstract}

days. Conclusion: While the frequency of psychological effects following blood donation identified by our study was comparable to others, the changes of the psychological state in our donors were traceable for a longer period than previously acknowledged.

(C) 2021 The Author(s) Published by S. Karger AG, Basel

\section{Introduction}

Maintaining a sufficient blood supply is a constant challenge for transfusion medicine. This is especially true for societies with an aging population $[1,2]$. The demographic change induces a shift from a younger to an older population, leading to a decline of eligible donors [35], while at the same time the increase in the elderly population will likely increase the demand for blood transfusion [4, 6-8]. A better understanding of the biopsychological and motivational factors of blood donation could help to establish new approaches to motivate and recruit blood donors. Many studies have addressed the motivational factors of blood donation [9-14] and adverse effects after blood donation [15-18]. However, only few investigations refer to positive psychological effects (in this study termed as "well-being") after blood donation [13, 19-26]. Previous qualitative studies provided some insights into the psychological effects after blood donation. Sojka and Sojka [22] identified in a retrospective study (with a self-administered questionnaire for 600 donors) the items "feeling of satisfaction," "more

\section{karger@karger.com} www.karger.com/tmh

Karger $\frac{1}{\%}$

BOPEN ACCESS
(C) 2021 The Author(s)

Published by S. Karger AG, Basel

This is an Open Access article licensed under the Creative Commons Attribution-NonCommercial-4.0 International License (CC BY-NC) (http://www.karger.com/Services/OpenAccessLicense), applicable to the online version of the article only. Usage and distribution for commercial purposes requires written permission. 
alert," and "feeling generally better" as positive effects of blood donation, while other authors found an increase in the well-being of blood donors $(n=108)$ in contrast to a control group $(n=108)$ emerging within $24 \mathrm{~h}$ after blood donation using the Multidimensional Mood Questionnaire (MDMQ) [21, 27]. This questionnaire was also completed by first-time whole blood donors in a study from Jansen et al. [20], who found only minor changes in the donors' well-being after blood donation. These changes in well-being were not associated with the return rate of donors. In a cross-sectional study from Denmark, $61 \%$ of blood donors experienced donation-related effects. The authors used a structured questionnaire that included both psychological (e.g., "energy") and physiological (e.g., "dizziness," "headache") symptoms [24]. Another study by van den Hurk et al. [28] used self-administered questionnaires to identify motivational factors and health characteristics in a representative cohort $(n=23,064)$ of the Dutch donor population. Positive effects ("feeling fit," "less headache") or negative effects ("lack of energy," "dizzy") after blood donation were reported by $4.6 \%$ and $13.9 \%$ of donors, respectively. Predonation symptoms ("headache," "lack of energy") occurred in $2.8 \%$ of donors. Beside psychological effects, other variables are well known to influence blood donors or may have a potential impact on donor well-being. In women, a low hemoglobin level has been described to be associated with increased vertigo [22] and dizziness [28] after blood donation, while 2 other studies reported an association between high pre-donation hemoglobin values and negative adverse effects in blood donors [18, 29]; in addition, low blood pressure increases the risk for vasovagal reactions after blood donation $[30,31]$ and other negative post-donation symptoms [28]. Furthermore, positive effects after blood donation were seen predominantly in donors with higher body mass index (BMI) [24, 28] and positive smoking status [24]. In this study, we present quantitative data on changes of the psychological state after blood donation using a newly established Well-Being Questionnaire (WBQ) and its application in a group of 100 regular blood donors with a follow-up of 8 weeks.

\section{Materials and Methods}

This cross-sectional prospective study consisted of 2 parts and was performed during 5 consecutive months before the corona virus epidemic at the Department of Transfusion Medicine at the University Medicine Greifswald (18,500 whole blood donations/ year). All participants were eligible for whole blood donation according to the national hemotherapy guidelines [30]. The study was approved by the local ethics committee of the University Medicine Greifswald and written informed consent was obtained from all participants.

\section{Study - Part 1}

Part 1 of this study was carried out to establish a questionnaire for the assessment of subjective changes in the well-being of whole blood donors after blood donation. Regular blood donors who had given at least 5 blood donations before were randomly asked in the waiting area of the blood donation center if they were willing to participate in an interview designed to yield information about psychological and physiological changes they had perceived after blood donation in the past. The interviews were conducted by the same interviewer in a face-to-face setting, recorded, and paper-based documented. The participants were asked whether they did "feel better, unchanged, or worse" after donating blood with regard to 4 time periods (the first few hours after donation, the first day after donation, 1-2 weeks after donation, and 3-4 weeks after donation). Then, the participants were encouraged to describe the psychological and/or physiological changes they had experienced during previous blood donations using their own words. The aim was to identify yet unrecognized effects or behavioral changes after blood donation. After that, the participants were asked preselected questions on effects after blood donation described in the literature. These questions addressed 10 common mood- and behavior-related dimensions (physical fitness; concentration; physical well-being; energy level; mood; alterations in the sleep behavior; satisfaction; creativity; resilience; spirit of initiative) and the dimension of observer-related changes (reaction or comments of family members or friends about the own behavioral changes). Questions were, for example: "Did you experience changes in your concentration after blood donation; e.g., while driving, reading, at work/housekeeping?" The recorded answers of participants were analyzed by the authors using a descriptive approach and frequencies of statements were counted. The most frequently mentioned changes after blood donation were then used to develop quantitative questions for the WBQ (Fig. 1) used in study part 2. Also, additional adjectives (e.g., "more or less alert" for the item concentration) were given to allow an easier understanding of the items (Fig. 1). To enable participants to give quantitative "translations" of changes in their well-being (per each item) in comparison to the situation before blood donation, the items were presented on a scale. The scale ranged from -5 to $+5 ;-5$ was the maximal negative decline on the item and +5 the maximal increase, while 0 indicated "no change" compared to the average well-being for the past 4-8 weeks before blood donation.

\section{Study - Part 2}

For part 2, regular whole blood donors with at least 3 previous blood donations were enrolled in the study before blood donation. Participants had to assess their well-being at 7 time points. During the first 3 time points, a paper-based version of the WBQ (Fig. 1) was filled in by participants in the donor clinic 15-30 min before the donation process, during the donation process, and 15-30 min after donation. Thereafter, the WBQ was applied by standardized telephone interviews with the participants at 5 different time points after blood donation (5-7 [mean 6] h after donation, 22-26 [mean 24] h after donation, 3 days after donation, 1 week after donation, and 8 weeks after donation). For easier understanding, participants received a version of the WBQ for their own use at home. All interviews were performed by the same interviewer to reduce variability. The answers of the participants were documented in a database by the interviewer. Donors of part 2 received a remuneration of EUR 15 after completed participation.

We also included additional variables known as potential confounders of well-being of blood donors: age, gender [18, 28, 30, 32], hemoglobin concentration [22], blood pressure [28, 33], and BMI [34]. 


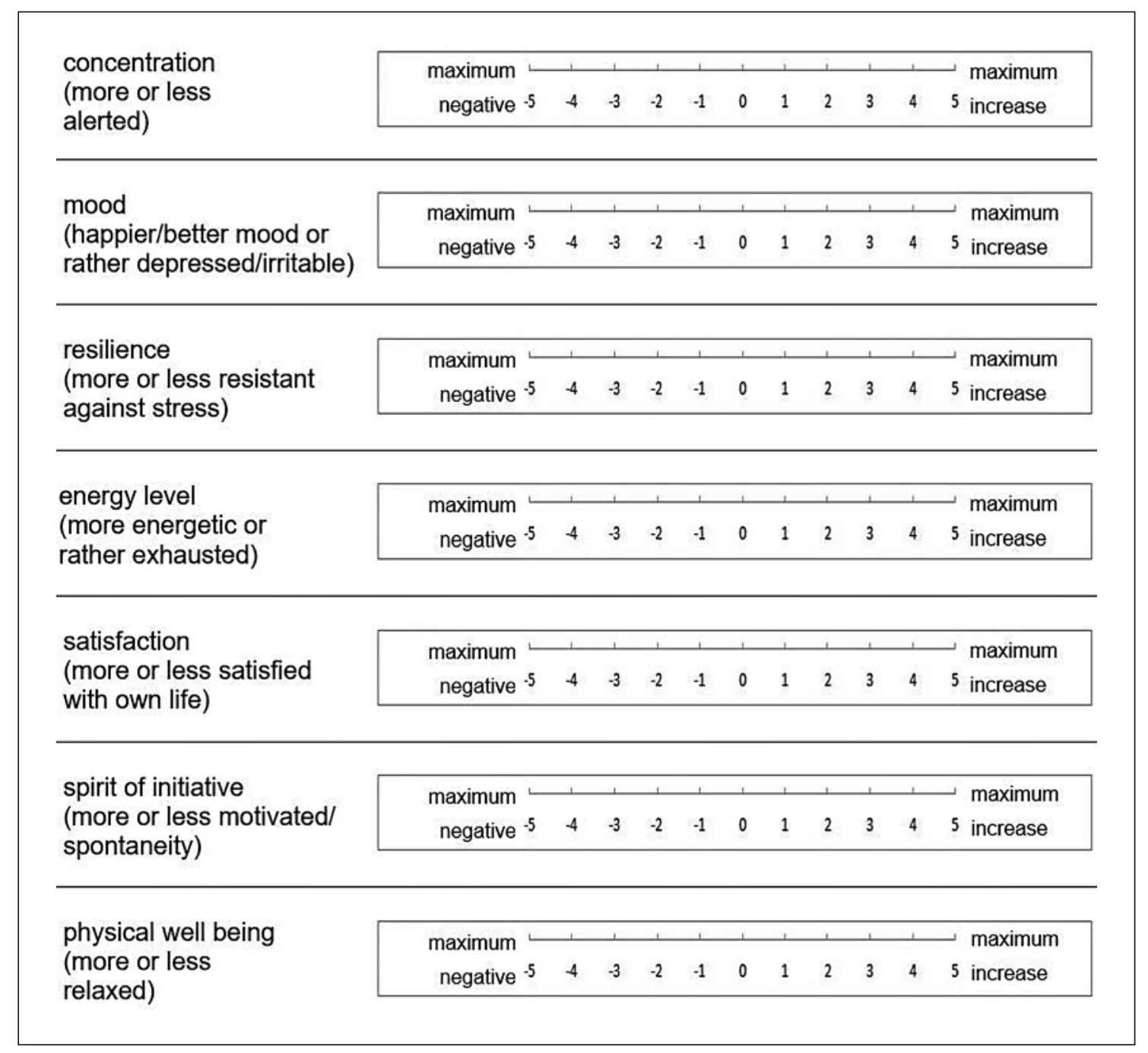

Fig. 1. Well-Being Questionnaire of study part 2. Instruction for participants: "Please fill out this questionnaire in the given scales. The 0 corresponds to the average level you would give yourself for the past $4-8$ weeks."

With the exception of blood pressure, the post-donation hemoglobin and BMI were not expected to change within 1 week after blood donation. We did not include items such as perceived needle pain, personal satisfaction, or personal interaction with the donor clinic staff, because these items had not been mentioned during the qualitative interviews by more than $25 \%$ of the regular blood donors. Furthermore, we had to keep the number of study questions within a manageable range during the multiple telephone interviews.

\section{Analysis of the WBQ}

To analyze the well-being after blood donation, the scores of the 7 items were summarized to the well-being score. At each time point, the well-being score could range from -35 (if all 7 items were judged "maximally negative") to +35 (if all 7 items were judged "maximally positive"). The well-being score at each time point was compared with the baseline score. We calculated the baseline score from the well-being score obtained 15-30 min before blood donation and the well-being score obtained 8 weeks after blood donation divided by 2 , as both the 15-30 min score and the 8 weeks score are very unlikely to be influenced by the blood donation. Then, we calculated a summary score for each donor. The summary score was obtained by subtracting the baseline score from the well-being scores obtained during the donation process, 15-30 min, 6 h, 24 h, 3 days, and 1 week after donation. By this approach, a delta value was obtained for each time point (well-being score at time point minus baseline score). For example, if the wellbeing score was 14 at 15-30 min before donation and 10 at 8 weeks after donation, the resulting baseline score was 12 . If at $24 \mathrm{~h}$ after donation the well-being score was 4 , this resulted in a delta of -8 . If the well-being score at 1 week after donation was 20 , this resulted in a delta of +8 . All delta variables of an individual participant were aggregated to the summary score. Next, we sketched out a diagram for the donors with their well-being scores over time. According to the individual curve of the well-being score, we assigned the donors to 1 of 3 different effect groups (positive effects, no effects, and negative effects after blood donation) by visual mapping. This was performed independently by all study authors. In the few cases of discrepant grouping, consensus was obtained by discussion. From the curves we then deducted the threshold values of the summary score for the 3 groups. 
Table 1. Characteristics of participants $(N=100)$

\begin{tabular}{|c|c|c|c|c|c|c|c|c|}
\hline & & \multicolumn{2}{|c|}{ Positive effects } & \multicolumn{2}{|c|}{ No effects } & \multicolumn{2}{|c|}{ Negative effects } & \multirow[t]{2}{*}{$p$ value } \\
\hline & & $n$ & mean $( \pm 2 \mathrm{SD})$ & $n$ & mean $( \pm 2 \mathrm{SD})$ & $n$ & mean $( \pm 2 \mathrm{SD})$ & \\
\hline \multirow[t]{2}{*}{ Sex } & Female & 9 & & 26 & & 12 & & \multirow[t]{2}{*}{0.463} \\
\hline & Male & 14 & & 27 & & 12 & & \\
\hline Age, years & & & $43.3( \pm 9.4)$ & & $42.9( \pm 11.7)$ & & $37.4( \pm 11.1)$ & 0.122 \\
\hline \multirow[t]{2}{*}{ Time of donation } & 9 a.m. to 1 p.m. & 8 & & 19 & & 7 & & \multirow{2}{*}{0.680} \\
\hline & 1 p.m. to 5 p.m. & 15 & & 34 & & 17 & & \\
\hline \multicolumn{2}{|c|}{ Systolic BP, mm Hg } & & $129.3( \pm 12.2)$ & & $128.2( \pm 11.4)$ & & $124.4( \pm 10.6)$ & 0.288 \\
\hline \multicolumn{2}{|c|}{ Diastolic BP, mm Hg } & & $81.7( \pm 6.7)$ & & $82.5( \pm 6.8)$ & & $76.9( \pm 7.6)$ & 0.006 \\
\hline \multicolumn{2}{|c|}{ Pre-donation hemoglobin concentration, g/dL } & & $14.65( \pm 1.45)$ & & $14.49( \pm 1.29)$ & & $14.49( \pm 1.13)$ & 0.606 \\
\hline \multicolumn{2}{|c|}{ Body mass index, $\mathrm{kg} / \mathrm{m}^{2}$} & & $26.6( \pm 3.1)$ & & $26.2( \pm 4.4)$ & & $25.7( \pm 4.0)$ & 0.753 \\
\hline \multicolumn{2}{|c|}{ Number of previous donations } & & $23.1( \pm 15.8)$ & & $19.7( \pm 13.5)$ & & $19.2( \pm 13.2)$ & 0.636 \\
\hline
\end{tabular}

$\mathrm{SD}$, standard deviation. BP, blood pressure.

Table 2. Cronbach's a score of the well-being measure with 7 items for the different time points

\begin{tabular}{lllllllll}
\hline Time point & $-30 \mathrm{~min}$ & $0 \mathrm{~min}$ & $30 \mathrm{~min}$ & $6 \mathrm{~h}$ & $24 \mathrm{~h}$ & $72 \mathrm{~h}$ & 1 week & 8 weeks \\
\hline$\alpha$ & 0.911 & 0.878 & 0.891 & 0.887 & 0.903 & 0.905 & 0.898 & 0.795 \\
\hline
\end{tabular}

\section{Statistics}

To summarize the different items of the WBQ to the well-being score, the internal consistency of the questionnaire was verified by Cronbach's a coefficient (function of the number of test items and the average inter-correlation among the items) for the different time points. This coefficient displays excellent internal consistency for a questionnaire with $\alpha \geq 0.9$, good internal consistency with 0.7 $\leq \alpha<0.9$, and acceptable internal consistency with $0.6 \leq \alpha<0.7$. The internal consistency for a questionnaire is usually considered to be insufficient at a Cronbach's $\alpha \leq 0.5$ [35].

Differences between the 3 groups according to the summary score and differences in the characteristics of the participants (age, gender, blood pressure, day time of blood donation, hemoglobin concentration) were calculated by Kruskal-Wallis test or MannWhitney U test; $p$ values $<0.05$ were regarded as significant. Values are expressed as mean values \pm standard deviation. Statistical analyses were computed by SPSS 21 for Windows.

\section{Results}

\section{Study - Part 1}

For the qualitative study, we enrolled 24 females (mean age $42.67 \pm 11.80$ years; range $24-59$ ) and 18 males (mean age $39.28 \pm 8.58$ years; range $25-50$ ).

The frequency of the commonly experienced psychological and physiological dimensions described by the participants of part 1 were counted numerically (absent/ present). Seven of the described dimensions were experienced after previous blood donations by at least $25 \%$ of the participants. These were mood (mentioned by $n=21$ blood donors), resilience $(n=20)$, spirit of initiative $(n=$ $12)$, concentration $(n=11)$, physical well-being $(n=24)$, energy level $(n=27)$, and satisfaction $(n=24)$. These dimensions were used to develop the WBQ for study part 2 (Fig. 1).

\section{Study - Part 2}

A total of 104 regular whole blood donors were enrolled, of whom 4 participants were excluded from further analysis because of missing data during the follow-up period. The characteristics of the 100 participants are shown in Table 1.

The Cronbach's a coefficient of the WBQ (Table 2) exceeded 0.7 (range 0.911-0.795) in all analyses, which indicates good internal consistency of the questionnaire across the investigation period or, in other words, the items in the WBQ all reliably measure the same latent variable (well-being).

According to the summary score, 23 donors showed positive effects on well-being after blood donation (aggregated summary score between 15 and 86.5), 53 donors showed no effects (summary score between -6.4 and 14.9 ), and 24 showed negative effects (summary score between -6.5 and -61.5 ). Representative examples for the individual curves of the well-being scores are shown in Figure $2 \mathrm{a}$ for a donor with positive effects, in Figure $2 \mathrm{~b}$ 


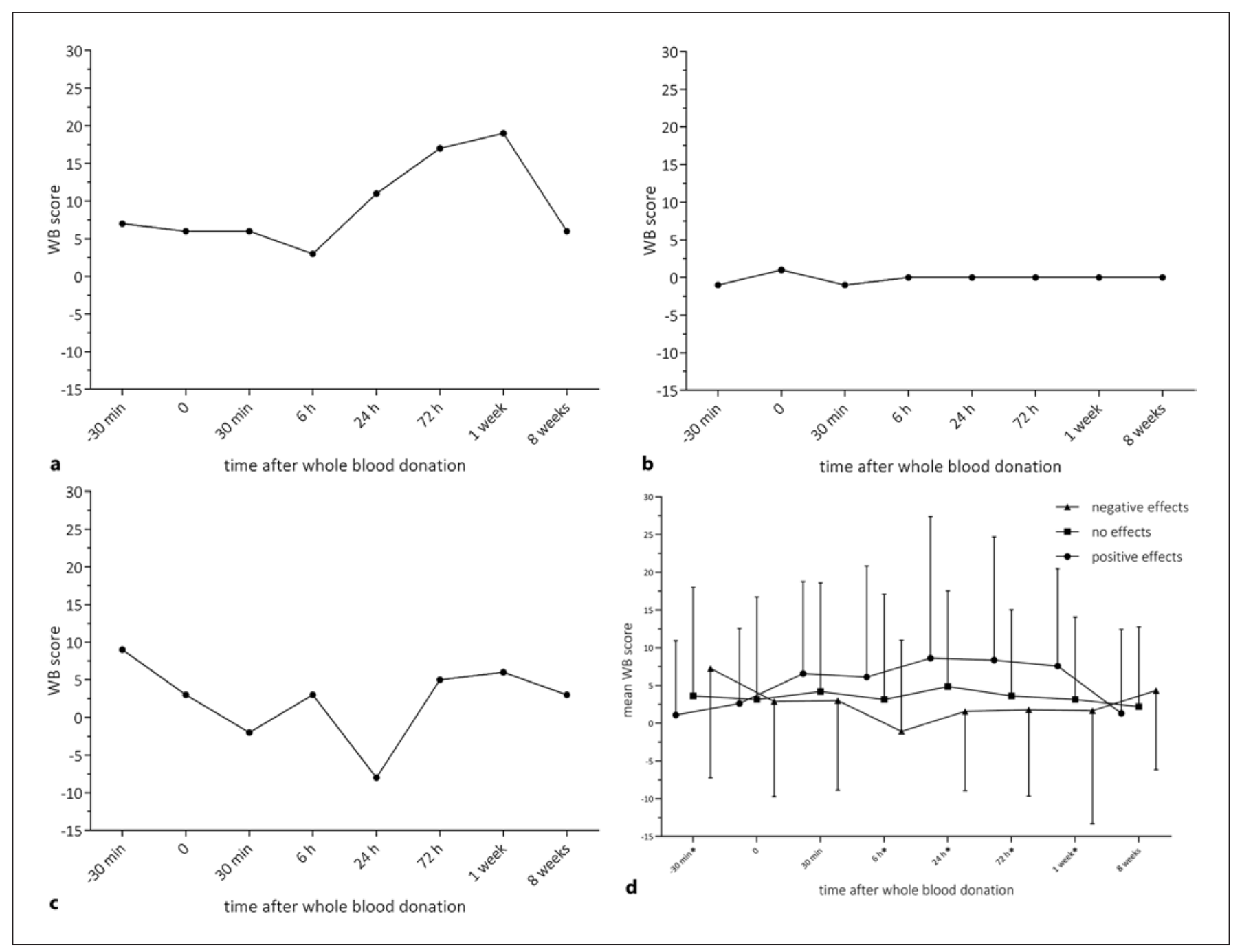

Fig. 2. a Individual curve of the well-being score over 8 weeks from a blood donor with positive effects after whole blood donation. b Individual curve of the well-being score over 8 weeks from a blood donor with no effects after whole blood donation. c Individual curve of the well-being score over 8 weeks from a blood donor with negative effects after whole blood donation. d Summary of the well-being scores of the 3 study groups $(n=100)$. The well-being scores of each participant were determined by adding all item scores obtained at a certain day. Of the 100 enrolled blood

donors, $n=23$ showed positive effects on their well-being (summary score between 15 and 86.5); $n=53$ donors showed no effects (summary score between -6.4 and 14.9); and $n=24$ donors showed negative effects (summary score between -6.5 and -61.5 ). The changes in the well-being scores over the observation period are given as mean $\pm 2 \mathrm{SD}$. ${ }^{*}$ indicates significant differences between the 3 groups. All data points refer to the time points given on the $x$-axis but are slightly moved in the graph for better readability.

for a donor without effects, and in Figure $2 c$ for a donor with negative effects after blood donation. The respective kinetics of the well-being scores in the different study groups are shown in Figure 2d. Donors with positive effects had a significantly higher well-being score compared to donors with no or negative effects already 15-30 min after blood donation (Mann-Witney $\mathrm{U}$ test; $p=$ 0.031). Furthermore, this higher well-being score of donors with positive effects lasted for a mean of 1 week after donation (Mann-Witney $\mathrm{U}$ test; $p=0.003$ ). In contrast, donors with negative effects had a significantly lower well-being score from $6 \mathrm{~h}$ to 3 days after donation com-

pared to donors with positive (Mann-Witney $\mathrm{U}$ test; $p=$ $0.04)$ or no effects $(p=0.014)$. Overall comparison of the 3 groups in a variance analysis showed significantly different well-being scores at the time points 15-30 min before blood donation (Kruskal-Wallis test; $p=0.016$ ), $6 \mathrm{~h}$ $(p=0.007), 24 \mathrm{~h}(p=0.003), 72 \mathrm{~h}(p=0.002)$, and 1 week $(p=0.0011)$ after blood donation.

Participants with low diastolic blood pressure $(<80$ $\mathrm{mm} \mathrm{Hg}$ ) prior donation had a higher risk for negative effects 15-30 min and $6 \mathrm{~h}$ after donation (Kruskal-Wallis test; $p=0.045$ and $p=0.014$ ) compared to blood donors with higher diastolic blood pressure $(\geq 80 \mathrm{~mm} \mathrm{Hg}$, odds 
ratio 5.32, 95\% confidence interval 1.83-15.49). Furthermore, younger blood donors ( $\leq 35$ years) had a lower wellbeing score compared to blood donors $>35$ years of age 6 $\mathrm{h}$ after donation (Kruskal-Wallis test; $p=0.006$ ).

Donors with positive, negative, and no effects after donation were similarly experienced with $23.1,19.2$, and 19.7 previous blood donations, respectively, before study participation (Kruskal-Wallis test; $p=0.636$ ). Thirty donors in the study had equal or fewer than 10 whole blood donations before study participation Positive, negative, or no effects occurred after blood donation in this study in 5, 17, and 8 donors, respectively. Another 40 participants were experienced donors with more than 20 donations. Positive, negative, or no effects were reported in this subgroup by 11,9 , and 20 donors, respectively.

\section{Discussion}

In this study, we show that blood donation influences the well-being of donors over several days. About $25 \%$ of donors experienced a positive effect with a mean duration of 1 week, $25 \%$ of donors experienced a negative effect with a mean duration of 3 days, while in half of the donors, blood donation had no effects on well-being.

These findings are very similar to those of other studies. Sojka and Sojka [22] identified donors with positive or negative and mixed positive and negative effects in $29 \%, 19 \%$, and $6 \%$ of donors, respectively, and observed no changes in well-being in about half of the donors [22]. Hinrichs et al. [21] also found positive (26.5\%) and negative (23.5\%) effects of blood donation on well-being in the same proportions of blood donors. The authors of the Danish Blood Donor Study determined in a cohort of 6,073 blood donors a prevalence of positive, negative, and mixed effects after blood donation with $18 \%, 29 \%$, and $14 \%$, respectively [24].

However, in a Dutch cohort study, positive and negative effects after blood donation were reported to occur in $4.6 \%$ and $13.9 \%$ of whole blood donors, respectively [28]. Differences in the prevalence of effects might be related to the questionnaire phrasing or the study design. While van den Hurk et al. [28] focused on physical effects ("feeling fit," "lack of energy," or "dizziness"), we asked donors for their well-being related to 7 different psychological dimensions. Together, 5 studies in Germany, the Netherlands, Denmark, and Sweden describe that at least 30\% of blood donors experience either psychological or physical effects after blood donation. This makes it rather likely that this finding is generalizable for a Middle and Northern European population.

Further evidence of a common effect is provided by the rather similar findings in the qualitative interviews we used in part 1 of our study to generate the questionnaire and in the studies by others using a self-administrated open-labelled questionnaire (identified effects, e.g." "feeling of satisfaction," "more alert," "feeling better," "tired," and "diminished physical capacity" [22] or "feeling fit," "lack of energy," and "dizziness" [24, 28]).

As outlined by others, the positive donation-related effects may be helpful in the recruitment of new donors and may motivate infrequent donors to donate more frequently $[21,22,24,28]$. While Jansen et al. [20] found that psychological effects had probably no impact on return rates of first-time donors, Suemnig et al. [13] showed that physical effects ("feeling physically better after donation") were a motivational factor to return to blood donation, especially for older donors ( $\geq 50$ years).

\section{Kinetics of the Changes in Well-Being}

The participants in our study reported that positive effects began within the first 30 min after blood donation. This is in concordance with other studies; for example, Hinrichs et al. [21] reported that positive effects occurred 15-30 min after blood donation; similar but only minor positive changes in the agitation level of first-time blood donors were noted in the first $30 \mathrm{~min}$ after blood donation in a study by Jansen et al. [20] who used the same questionnaire as Hinrichs et al. [21]. A higher elation and decreased anxiety score of donors up to $60 \mathrm{~min}$ after donation were seen in study by Zillmer et al. [26]; and Sojka and Sojka [22], who used a retrospective approach in their study, report the putative onset of effects within $1 \mathrm{~h}$ after donation. This study also found that positive effects last longer than negative effects [20], which is similar to our results, where the positive changes in well-being lasted for 1 week and the negative effects for 3 days.

\section{Other Cofactors}

The number of previous blood donations had no major impact on changes in well-being, in concordance with results from other investigators $[21,22,28]$. Notably, the study by van den Hurk et al. [28] found neither positive nor negative effects associated with the number of prior blood donations in a representative cohort of over 23,000 blood donors, with most participants having experienced a total of 5 or more donations [28]. Interestingly, Sojka and Sojka [22], who included donors with fewer than 5 donations, found no differences in the distribution of effects according to the number of previous blood donations [22]. However, as we only enrolled donors with at least 3 previous blood donations (the vast majority with at least 5 previous blood donations), we do not know whether this might be different in donors with only 1 or 2 previous blood donations. Also, our observation that younger donors ( $\leq 35$ years) had a significantly lower well-being score $6 \mathrm{~h}$ after blood donation is comparable to results of 2 cohort studies from Sweden [22] and Denmark [24], 
who described more negative psychological effects in younger donors than in older donors. This might in part be related to an increased rate of vasovagal reactions in younger blood donors $[30,31]$, especially if they are unexperienced donors. While Teglkamp et al. [24] found positive effects associated with age, we and others [21, 22, 28] could not generally confirm this association.

In contrast to other studies [21,22,28], we observed no association between donation-induced changes in the donors' well-being and the pre-donation hemoglobin concentration. We also found no significant differences in well-being depending on gender or BMI, while others observed a higher rate of negative effects after whole blood donation in females compared to males $[21,24,28]$ and a correlation of a higher BMI with negative symptoms before ("lack of energy," "headache") [26] or positive symptoms ("alleviated headache") after blood donation [24]. Finally, van den Hurk et al. [28] and the present study suggest that donors with a lower diastolic blood pressure experience more negative effects after blood donation in comparison to donors with a higher diastolic blood pressure, while a systematic review found no evidence of an increased risk of adverse physical effects after blood donation in subjects with hypotension prior to the donation [36].

\section{Study Limitations}

Due to the single-center design of our study, we cannot exclude that specific local factors had an effect on the emotional reactions of the donors after blood donation, for example, personal interaction between the donor and staff of the donor clinic. Furthermore, the results may not be transferable to the donor cohort of other blood donor services due to the large number of young participants in our study, reflecting the location of our donor center within a small university city. Future studies should be performed in a donor population of a large national or regional blood service. The study should enroll young and older participants to confirm our results, ideally in a prospective manner over at least 1 week after donation.

We aimed to generate a set of 7 questions relevant to blood donation based on study part 1 with qualitative interviews. However, we did not extensively validate the selected items using different cohorts independent of blood donation. The quantitative approach to the questions allowed us to summarize the items to a total score. However, despite controlling with Cronbach's a test for consistency we cannot exclude that we lost some information provided by the individual questions.

We summarized the scores 15-30 min before donation and 8 weeks after donation as baseline scores. Potentially, the values obtained shortly before blood donation had been influenced by some form of anxiety or headache as shown by van den Hurk et al. [28] and Teglkamp et al. [24]. However, as outlined above, only "trained" regular blood donors with at least 3 previous donations were enrolled in study part 2 , which makes the impact of anxiety factors probably low.

Our study is a correlational study, hence any observed variability in well-being ratings over time may reflect life experiences that have nothing to do with blood donation (e.g., serious family event; car accident). Controlling nonblood donation variables by a matched population of individuals who did not donate blood was not feasible in the present study. Furthermore, we analyzed the change in variables over 8 weeks, which should have levelled out some of the non-blood donation related effects which may have impacted well-being.

In summary, the frequencies of positive and negative effects in donors following blood donation seem to be comparable among at least 3 different studies, including the present study, with about $25 \%$ of donors experiencing positive psychological effects after blood donation and about the same percentage of donors experiencing negative psychological effects after blood donation. Given these rather reproducible findings, further studies should assess whether physiological mechanisms impact these changes in psychological factors to identify potential biomolecular changes induced by blood donation.

\section{Conflict of Interest Statement}

The authors declare that they have no conflicts of interest relevant to the manuscript submitted to Transfusion Medicine and Hemotherapy.

\section{Statement of Ethics}

This study was conducted ethically in accordance with the World Medical Association Declaration of Helsinki. The study was approved by the local ethics committee of the University Medicine Greifswald and written informed consent was obtained from all participants. All authors had full access to all data including all statistical reports and tables used in the manuscript.

\section{Funding Sources}

We acknowledge support for the Article Processing Charge from the DFG (German Research Foundation, 393148499) and the Open Access Publication Fund of the University of Greifswald.

\section{Author Contributions}

M.E., H.J.G., and A.G. conceived and designed the study. M.E., A.S., U.A., and A.G. acquired, analyzed, or interpreted the data, drafted the article or provided critical revision for important intellectual content, and provided final approval of the version to be published. All authors are accountable for all aspects of the work in ensuring that questions related to the accuracy or integrity of any part of the article are appropriately investigated and resolved. 


\section{References}

1 Greinacher A, Fendrich K, Hoffmann W. Demographic Changes: The Impact for Safe Blood Supply. Transfus Med Hemother. 2010; 37:141-8.

2 Volken T, Buser A, Castelli D, Fontana S, Frey BM, Rüsges-Wolter I, et al. Red blood cell use in Switzerland: trends and demographic challenges. Blood Transfus. 2018;16:73-82.

3 Ehling M, Pötzsch O. Demographic Changes in Germany up to 2060 - Consequences for Blood Donation. Transfus Med Hemother. 2010;37:131-9.

4 Schönborn L, Weitmann K, Greger N, Kiefel V, Hoffmann W, Greinacher A. Longitudinal Changes in the Blood Supply and Demand in North-East-Germany 2005-2015. Transfus Med Hemother. 2017;44:224-31.

5 Müller-Steinhardt M, Weidmann C, Klüter $\mathrm{H}$. Changes in the Whole Blood Donor Population in South-West Germany: 2010 versus 2016. Transfus Med Hemother. 2017;44:21723.

6 Barr PJ, Donnelly M, Morris K, Parker M, Cardwell C, Bailie KE. The epidemiology of red cell transfusion. Vox Sang. 2010;99:23950.

7 Geißler RG, Franz D, Buddendick H, Krakowitzky P, Bunzemeier H, Roeder N, et al. Retrospective Analysis of the Blood Component Utilization in a University Hospital of Maximum Medical Care. Transfus Med Hemother. 2012;39:129-38.

8 Ali A, Auvinen MK, Rautonen J. The aging population poses a global challenge for blood services. Transfusion. 2010;50:584-8.

9 Masser BM, White KM, Hyde MK, Terry DJ. The psychology of blood donation: current research and future directions. Transfus Med Rev. 2008;22:215-33.

10 Bednall TC, Bove LL, Cheetham A, Murray AL. A systematic review and meta-analysis of antecedents of blood donation behavior and intentions. Soc Sci Med. 2013;96:86-94.

11 Bednall TC, Bove LL. Donating blood: a metaanalytic review of self-reported motivators and deterrents. Transfus Med Rev. 2011;25 317-34.

12 Godin G, Vézina-Im LA, Bélanger-Gravel A, Amireault S. Efficacy of interventions promoting blood donation: a systematic review. Transfus Med Rev. 2012;26:224-e6.

13 Suemnig A, Konerding U, Hron G, Lubenow $\mathrm{N}$, Alpen U, Hoffmann W, et al. Motivational factors for blood donation in first-time do- nors and repeat donors: a cross-sectional study in West Pomerania. Transfus Med. 2017;27:413-20.

14 Ferguson E, Taylor M, Keatley D, Flynn N, Lawrence C. Blood donors' helping behavior is driven by warm glow: more evidence for the blood donor benevolence hypothesis. Transfusion. 2012;52:2189-200.

15 Amrein K, Valentin A, Lanzer G, Drexler C. Adverse events and safety issues in blood donation - a comprehensive review. Blood Rev. 2012;26:33-42.

16 Newman BH. Donor reactions and injuries from whole blood donation. Transfus Med Rev. 1997;11:64-75.

17 Riga A, Sapey T, Bacanu M, Py J-Y, Dehaut F. Blood donors - Serious adverse reactions (SAR) 2010-2014 EFS Châteauroux, France. Transfus Clin Biol. 2015;22:62-5.

18 Wiersum-Osselton JC, Marijt-van der Kreek T, Brand A, Veldhuizen I, van der Bom JG, de Kort W. Risk factors for complications in donors at first and repeat whole blood donation: a cohort study with assessment of the impact on donor return. Blood Transfus. 2014; 12(Suppl 1):s28-36.

19 Uma S, Arun R, Arumugam P. The knowledge, attitude and practice towards blood donation among voluntary blood donors in Chennai, India. J Clin Diagn Res. 2013;7: 1043-6.

20 Jansen P, Sümnig A, Esefeld M, Greffin K, Kaderali L, Greinacher A. Well-being and return rate of first-time whole blood donors. Vox Sang. 2019;114:154-61.

21 Hinrichs A, Picker SM, Schneider A, Lefering R, Neugebauer EA, Gathof BS. Effect of blood donation on well-being of blood donors. Transfus Med. 2008;18:40-8.

22 Nilsson Sojka B, Sojka P. The blood-donation experience: perceived physical, psychological and social impact of blood donation on the donor. Vox Sang. 2003;84:120-8.

23 Piliavin JA, Callero PL, Evans DE. Addiction to altruism? Opponent-process theory and habitual blood donation. J Pers Soc Psychol. 1982;43(6):1200-13.

24 Teglkamp J, Handgaard L, Hansen T, Pedersen OB, Rigas AS, Mikkelsen S, et al. The donors perceived positive and negative effects of blood donation. Transfusion. 2020;60:553-60.

25 van den Hurk K, Zalpuri S, Prinsze FJ, Merz EM, de Kort WLAM. Associations of health status with subsequent blood donor behav- ior - An alternative perspective on the Healthy Donor Effect from Donor InSight. PLoS One. 2017;12:e0186662.

26 Zillmer EA, Glidden RA, Honaker LM, Meyer JD. Mood states in the volunteer blood donor. Transfusion. 1989;29:27-30.

27 Steyer R. Der mehrdimensionale Befindlichkeitsfragebogen: MDBF. Göttingen: Hogrefe, Verlag für Psychologie; 1997.

28 van den Hurk K, Peffer K, Habets K, Atsma F, Pasker-de Jong PCM, van Noord PAH, et al. Blood donors' physical characteristics are associated with pre- and post-donation symptoms - Donor InSight. Blood Transfus. 2017; 15:405-12.

29 Gonçalez TT, Sabino EC, Schlumpf KS, Wright DJ, Leao S, Sampaio D, et al. Vasovagal reactions in whole blood donors at three REDS-II blood centers in Brazil. Transfusion. 2012;52:1070-8.

30 Tondon R, Pandey P, Chaudhary R. Vasovagal reactions in 'at risk' donors: a univariate analysis of effect of age and weight on the grade of donor reactions. Transfus Apher Sci. 2008;39:95-9.

31 Trouern-Trend JJ, Cable RG, Badon SJ, Newman BH, Popovsky MA. A case-controlled multicenter study of vasovagal reactions in blood donors: influence of sex, age, donation status, weight, blood pressure, and pulse. Transfusion. 1999;39:316-20.

32 Wiltbank TB, Giordano GF, Kamel H, Tomasulo $\mathrm{P}$, Custer B. Faint and prefaint reactions in whole-blood donors: an analysis of predonation measurements and their predictive value. Transfusion. 2008;48:1799-808.

33 France CR, Rader A, Carlson B. Donors who react may not come back: analysis of repeat donation as a function of phlebotomist ratings of vasovagal reactions. Transfus Apher Sci. 2005;33:99-106.

34 Wiltink J, Michal M, Wild PS, Zwiener I, Blettner M, Münzel T, et al. Associations between depression and different measures of obesity (BMI, WC, WHtR, WHR). BMC Psychiatry. 2013;13:223.

35 Peterson RA. A Meta-Analysis of Cronbach's Coefficient Alpha. J Consum Res. 1994;21(2): 381.

36 Pauwels NS, Cusack L, De Buck E, Compernolle V, Vandekerckhove P. The effect of predonation hypotension on whole blood donor adverse reactions: a systematic review. J Am Soc Hypertens. 2014;8:429-36. 